\title{
Severe ocular phenotypes in Rbp4-deficient mice in the C57BL/6 genetic background
}

\author{
Jingling Shen ${ }^{1,8}$, Dan Shi ${ }^{2,8}$, Tomohiro Suzuki ${ }^{3}$, Zunping Xia ${ }^{4}$, Hanli Zhang ${ }^{2}$, Kimi Araki ${ }^{5}$, Shigeharu Wakana ${ }^{4}$, \\ Naoki Takeda ${ }^{5}$, Ken-ichi Yamamura ${ }^{6}$, Shoude $\mathrm{Jin}^{7}$ and Zhenghua Li, ${ }^{1,6}$
}

Retinol-binding protein 4 (RBP4) is a specific carrier for retinol in the blood. In hepatocytes, newly synthesized RBP4 associates with retinol and transthyretin and is secreted into the blood. The ternary transthyretin-RBP4-retinol complex transports retinol in the circulation and delivers it to target tissues. Rbp4-deficient mice in a mixed genetic background (129xC57BL/6J) have decreased sensitivity to light in the b-wave amplitude on electroretinogram. Sensitivity progressively improves and approaches that of wild-type mice at 24 weeks of age. In the present study, we produced Rbp4-deficient mice in the C57BL/6 genetic background. These mice displayed more severe phenotypes. They had decreased a- and b-wave amplitudes on electroretinograms. In accordance with these abnormalities, we found structural changes in these mice, such as loss of the peripheral choroid and photoreceptor layer in the peripheral retinas. In the central retinas, the distance between the inner limiting membrane and the outer plexiform layer was much shorter with fewer ganglion cells and fewer synapses in the inner plexiform layer. Furthermore, ocular developmental defects of retinal depigmentation, optic disc abnormality, and persistent hyaloid artery were also observed. All these abnormalities had not recovered even at 40 weeks of age. Our Rbp4-deficient mice accumulated retinol in the liver but it was undetectable in the serum, indicating an inverse relation between serum and liver retinol levels. Our results suggest that RBP4 is critical for the mobilization of retinol from hepatic storage pools, and that such mobilization is necessary for ocular development and visual function.

Laboratory Investigation (2016) 96, 680-691; doi:10.1038/labinvest.2016.39; published online 14 March 2016

\section{Introduction}

Retinol-binding protein 4 (RBP4) is a specific carrier for retinol in the blood. In hepatocytes, newly synthesized RBP4 associates with retinol and transthyretin (TTR) in the endoplasmic reticulum and is then secreted into the blood. $^{1-5}$ Thus, RBP4 is essential for the mobilization of retinol from liver stores. Binding to TTR (a tetramer of MW 55000 ) prevents renal filtration of RBP4 (MW 21000 ). ${ }^{2}$

In humans, genome-wide association studies revealed new susceptibility loci for eye diseases, such as refractive error including myopia. ${ }^{6-8}$ Over 450 inbred strains of mice have been described, ${ }^{9}$ providing a wealth of different genotypes and phenotypes for studying human diseases.
Keane et $a l^{10}$ reported the genome sequences of 17 inbred strains of laboratory mice and identified almost 10 times more variants than was previously known. Many of them result in significant tissue-specific expression bias and phenotypic variation. ${ }^{10,11} \mathrm{C} 57 \mathrm{BL} / 6 \mathrm{~J}$ mice are susceptible to high-fat diet-induced type 2 diabetes. ${ }^{12}$ JF1 mice are especially sensitive to high-fat diet-induced diabetes and obesity, whereas MSM/Ms mice are resistant. ${ }^{13} \mathrm{We}^{14}$ showed that the severity of acute pancreatitis experimentally induced by cerulein was highest in $\mathrm{C} 3 \mathrm{H} / \mathrm{HeJ}$ and $\mathrm{CBA} / \mathrm{J}$, moderate in $\mathrm{BALB} / \mathrm{c}$, and mildest in C57BL/6J and JF1 mice.

To analyze the relationship between sequence variations and phenotypic variations, it is essential to create transgenic

\footnotetext{
'Department of Histology and Embryology, Harbin Medical University, Harbin, China; ${ }^{2}$ Department of Anesthesia, Daqing Oil field General Hospital, Daqing, China; ${ }^{3}$ Technology and Development Team for Mouse Phenotype Analysis, RIKEN BioResource Center, Tsukuba, Japan; ${ }^{4}$ Department of Anesthesia, Fifth Hospital of Harbin, Harbin, China; ${ }^{5}$ Division of Developmental Genetics, Institute of Resource Development and Analysis, Kumamoto University, Kumamoto, Japan; ${ }^{6}$ Yamamura Project Laboratory, Institute of Resource Development and Analysis, Kumamoto University, Kumamoto, Japan and ${ }^{7}$ Division of Respiratory Disease, The Fourth Affiliated Hospital of Harbin Medical University, Harbin, China

Correspondence: Dr S Jin, MD, PhD, Division of Respiratory Disease, The Fourth Affiliated Hospital of Harbin Medical University, 37 Yiyuan Street, Nangang District, Harbin 150001, China or Dr Z Li, MD, PhD, Department of Histology and Embryology, Harbin Medical University, 194 Xuefu Road, Nangang District, Harbin 150081, China. E-mail: jinshoude@163.com or liseika@kumamoto-u.ac.jp

${ }^{8}$ These authors contributed equally to this work.

Received 6 August 2015; revised 1 February 2016; accepted 4 February 2016
} 


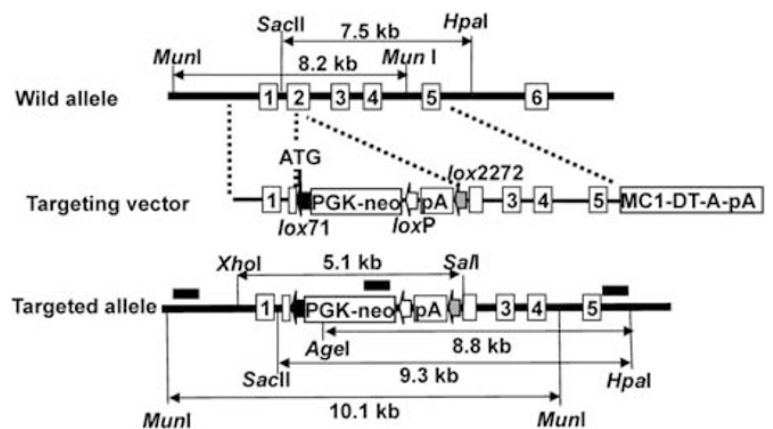

Figure 1 Targeting vector construction. Homologous recombination between the wild-type allele and the targeting vector produced a targeted null allele carrying the PGK-neo gene flanked by lox71 and loxP. MC1-DT-A-pA: a diphtheria toxin A (DT-A) fragment with an MC1 promoter.

or knockout mice with a defined genetic background. So far, Quadro et $\mathrm{al}^{15}$ generated Rbp4-deficient mice by using 129/Sv embryonic stem (ES) cells and mated these chimeric mice with C57BL/6J to obtain Rbp4-deficient mice in a mixed genetic background $(129 \times \mathrm{C} 57 \mathrm{BL} / 6 \mathrm{~J})$. Although these mice showed impaired visual function, it progressively improved by 24 weeks of age.

Here, we created targeted null $R b p 4$ allele mice in the C57BL/6 background and showed that these $R b p 4^{-1-}$ mice had much more severe ocular phenotypes in terms of impaired visual function, and structural and developmental defects.

\section{MATERIALS AND METHODS Construction of Targeting Vectors and Isolation of Targeted ES Cells}

The construction of targeting vectors and isolation of targeted ES cells were carried out as described previously, ${ }^{16}$ except for the use of a $2.8-\mathrm{kb} \quad 5^{\prime} \quad R b p 4$ homologous region upstream of the ATG codon and a 6.7-kb $3^{\prime} R b p 4$ homologous region downstream of the ATG codon (Figure 1).

\section{Southern Blot Analyses for Isolation of Targeted ES Cell Clones}

So far, all ES cell lines have been established from C57BL/6N mice, but not from C57BL/6J. Mattapallil et al ${ }^{17}$ showed that a series of commercially available ES cells of C57BL/6N origin and $\mathrm{C} 57 \mathrm{BL} / 6 \mathrm{~N}$ mouse lines used to generate $\mathrm{ES}$ cells contained the $r d 8$ mutation in the $C r b 1$ gene. This mutation results in a form of retinal dysplasia and degeneration. ${ }^{3}$ To establish an Rbp4-deficient mouse in the C57BL/6 background, we used TT2 ES cells originated from an F1 embryo between C57BL/6N and CBA/JN strains. $^{18}$

Targeted ES cell clones were confirmed by Southern blot analyses as described previously. ${ }^{16}$ For detection of homologous recombination in the $5^{\prime}$ region, DNA from ES cells was digested with XhoI/SalI or MunI and the targeted allele was detected with a neo probe and a $5^{\prime}$ probe; for detection of homologous recombination in the $3^{\prime}$ region, AgeI/HpaI or SacII/HpaI digestion and a neo probe and $3^{\prime}$ probe were used, respectively (Supplementary Figure S1).

\section{Generation of Rbp4-Knockout Mice}

Chimeric mice were produced by aggregation of ES cells with eight-cell embryos from ICR mice as described previously. ${ }^{16}$ ICR originated from the CD1 strain at the Institute of Cancer Research (Philadelphia, PA, USA). ICR is an albino mouse kept in closed colony at Clea (Tokyo, Japan; http://www.cleajapan.com/en/index.html). To exclude the $r d 8$ mutation, chimeric male mice were mated to C57BL/6J females (Clea) to obtain the $R b p 4^{-/+}$offspring. These $\mathrm{F} 1$ mice were backcrossed to C57BL/6J for a further six generations. At the second and sixth generation, we carried out markerassisted breeding by using 123 polymorphic markers (111 markers on autosomes, 8 markers on $\mathrm{X}$ chromosome and 4 markers on $\mathrm{Y}$ chromosome). These markers can distinguish between C57BL/6 and CBA/JN alleles. These polymorphic markers were distributed evenly throughout the genome and detected by using a high-speed genetic profiling system (384-well plate-based TaqMan assay method) (Japan Mouse Clinic; http://ja.brc.riken.jp/lab/jmc/mouse clinic/en/assistive/index.html) and mice with C57BL/6 alleles at most loci were selected for breeding. All mice used in our experiments were at the seventh generation and had C57BL/ 6-homozygous alleles at all 123 polymorphic loci. Mice were fed a CE-2 diet (Clea), which contained $1515 \mathrm{IU}$ retinol/100 g $(0.454 \mathrm{mg} / 100 \mathrm{~g})$. To avoid possible sex-related differences, only males were used in all analyses. All experiments were performed in accordance with the Declaration of Helsinki and were approved by the Kumamoto University Ethics Committee for Animal Experiments.

\section{Northern Blot, Semiquantitative RT-PCR, and qRT-PCR Analyses}

Northern blot and semiquantitative reverse transcriptionpolymerase chain reaction (RT-PCR) analyses were carried out as described by Zhao et al ${ }^{16}$ and Li et al, ${ }^{19}$ respectively. The nucleotide sequence identity between the mouse and human $R b p 4$ coding regions is $\sim 87 \%$. The primer set suitable for human and mouse RBP4 coding regions was as follows: sense (mhRBP4-F), 5'-GAGTCAAGGAGAACTTCGAC-3'; antisense (mhRBP4-e4R), 5'-CAGTACTTCATCTTGAACTT GG-3'; the PCR product was expected to be 244 -bp long. The primer set used for detection of the Hprt mRNA was as follows: sense, 5'-CACAGGACTAGAACACCTGC-3'; antisense, 5'-GCTGGTGAAAAGGACCTCT-3'; the PCR product was expected to be 238-bp long. PCR products were separated on $1.5 \%$ agarose gels and stained with ethidium bromide. Quantitative RT-PCR (qRT-PCR) was performed with an Applied Biosystems 7500 Real-Time PCR system (Applied 

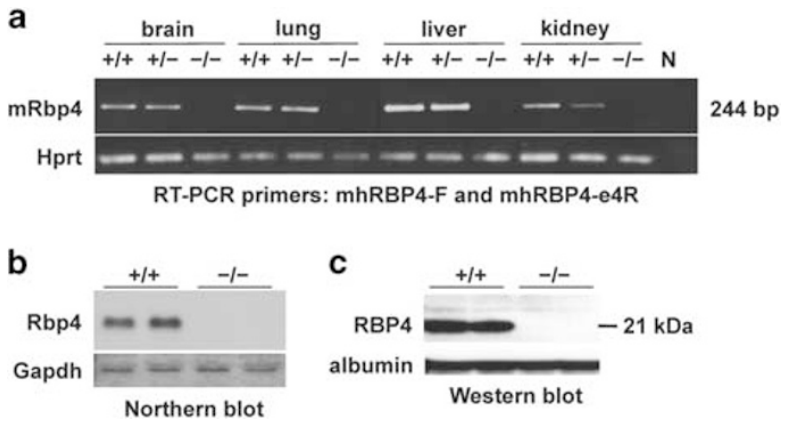

Figure 2 Confirmation of null mutation. (a) Semiquantitative reverse transcription-polymerase chain reaction (RT-PCR) analysis. The expected 244-bp band was detected in tissues from $\mathrm{Rbp}^{+/+}$and $\mathrm{Rbp}^{+/-}$mice but not in those from $R b p 4^{-1-}$ mice. (b) Northern and western blot analyses. No Rbp4 mRNA or RBP4 protein was detected in the liver or serum, respectively, from $R_{b p 4^{-1-}}$ mice.

Biosystems, Foster City, CA, USA). Reactions were carried out under the following conditions: $1 \mathrm{~min}$ at $95^{\circ} \mathrm{C}$, followed by 40 cycles of $15 \mathrm{~s}$ at $95^{\circ} \mathrm{C}$ and $1 \mathrm{~min}$ at $60^{\circ} \mathrm{C}$. Quantified values were normalized against the input determined by expression of the housekeeping gene Hprt.

\section{Western Blot Analysis}

Mouse serum ( $6 \mu$ l diluted 1:50 in $0.9 \% \mathrm{NaCl}$ per lane) was separated in a $12 \%$ polyacrylamide gel and transferred to an Immobilon polyvinylidene difluoride membrane (Millipore, Billerica, MA, USA). The following primary antibodies were used: rabbit anti-human RBP4 antibody (1:1000; PTG, Chicago, IL, USA) and rabbit anti-bovine serum albumin antibody (1:1500; Upstate, New York, NY, USA). Anti-rabbit immunoglobulin $\mathrm{G}$ conjugated with horseradish peroxidase (Amersham Biosciences, Piscataway, NJ, USA) was used for detection. Band intensities were quantified by densitometry and the ImageJ ver. 1.38 software (http://rsb.info.nih.gov/ij/ docs/index.html).

\section{Functional Analysis of the Retina}

Electroretinograms (ERGs) were performed as described previously. ${ }^{20}$ Left eyes of $R b p 4^{+/+}$and $R b p 4^{-1-}$ mice at 7 , 14,24 , and 40 weeks of age were used. Mice were dark-adapted overnight and anesthetized with a mixture of ketamine $(80 \mathrm{mg} / \mathrm{kg}$; Daiichi Sankyo Propharma, Tokyo, Japan) and xylazine (16 mg/kg; Bayer Medical, Tokyo, Japan) under dim red light. Pupils were dilated with Mydrin-P ophthalmic solution (1:5 dilution) and corneas were anesthetized with $0.4 \%$ oxybuprocaine hydrochloride (Santen Pharmaceutical, Tokyo, Japan). Stimulus presentation was programmed and data acquisition was performed with the data recording and analysis software (Scope v.3.7; ADInstruments, Bella Vista, Australia). Scotopic ERG responses to single flash presentations (10-ms duration) at three increasing intensities $\left(0.4,1.2\right.$, and $\left.2.0 \log \mathrm{cd} \mathrm{s} / \mathrm{m}^{2}\right)$ were recorded. Triplicate responses were computer-averaged for each flash condition. The time intervals between flashes were 10,30 , and $60 \mathrm{~s}$ for intensities of $0.4,1.2$, and $2.0 \log \mathrm{cd} \mathrm{s} / \mathrm{m}^{2}$, respectively. The amplitude and implicit time of the a-wave were measured from the prestimulus baseline to the a-wave trough. The amplitude and implicit time of the b-wave were measured from the a-wave trough to the peak of the b-wave or, if the a-wave was not measurable, from the prestimulus baseline.

\section{Retinal Histology}

After the ERG was performed, mice (40 weeks of age) were anesthetized with an intraperitoneal injection of pentobarbital sodium ( $90 \mathrm{mg} / \mathrm{kg}$; Kyoritsu Seiyaku, Tokyo, Japan). Left eyes were enucleated and fixed overnight in $2.0 \%$ paraformaldehyde $/ 2.0 \%$ glutaraldehyde in $0.1 \mathrm{M}$ cacodylic acid, $\mathrm{pH}$ 7.4. The eye was dehydrated by immersion in a series of increasing ethanol concentrations. After three changes of xylene, the eyes were embedded in paraffin wax. A mid-horizontal series of sections $(3-\mu \mathrm{m}$-thick) through the optic nerve head was prepared. Sections were stained with hematoxylin and eosin (Muto Pure Chemicals, Tokyo, Japan) and viewed with an AX80 microscope (Olympus, Tokyo, Japan).

\section{Measurement of Retinal Properties}

The number of cells in the ganglion cell layer and the thickness of the inner nuclear layer (INL), outer nuclear layer $(\mathrm{ONL})$, and photoreceptor outer segment layer (OSL) were measured by using light microscopy. The measurements were performed at the points where the ratios of the distance from the optic disc to the point to the distance from the optic disc to peripheral end of the retina were 0.2 and $0.8 .^{20,21}$ The number of cells in the ganglion cell layer was estimated at the central retina $(220 \mu \mathrm{m}$ from the optic nerve head) and peripheral retina $(220 \mu \mathrm{m}$ from the peripheral edge $){ }^{21}$ The distance from the retinal surface to the end of the photoreceptor outer segments was measured as the retinal thickness. The distance from the peripheral edge of the retina to the central end was measured as the distance from the central to peripheral retina. The values recorded for each parameter for the nasal and temporal sides were averaged to obtain a single value for each eye. For consistency, only retinal sections with optic nerve stumps were used.

\section{Transmission Electron Microscopy}

Mice at 40 weeks of age were used for this study. The eyes were fixed as described above at $4{ }^{\circ} \mathrm{C}$ overnight, washed three times with $0.1 \mathrm{M}$ cacodylate buffer for $30 \mathrm{~min}$ each, and postfixed with $2 \%$ osmium tetroxide in $0.1 \mathrm{M}$ cacodylate buffer at $4{ }^{\circ} \mathrm{C}$ for $2 \mathrm{~h}$. The samples were then dehydrated in a graded ethanol series: 50 and $70 \%$ for $30 \mathrm{~min}$ each at $4{ }^{\circ} \mathrm{C}$, $90 \%$ for $30 \mathrm{~min}$ at room temperature, three changes of $100 \%$ for $30 \mathrm{~min}$ each at room temperature, and 100\% ethanol overnight at room temperature. The samples were infiltrated with propylene oxide (PO) two times for $30 \mathrm{~min}$ each and were placed into a 70:30 mixture of $\mathrm{PO}$ and 


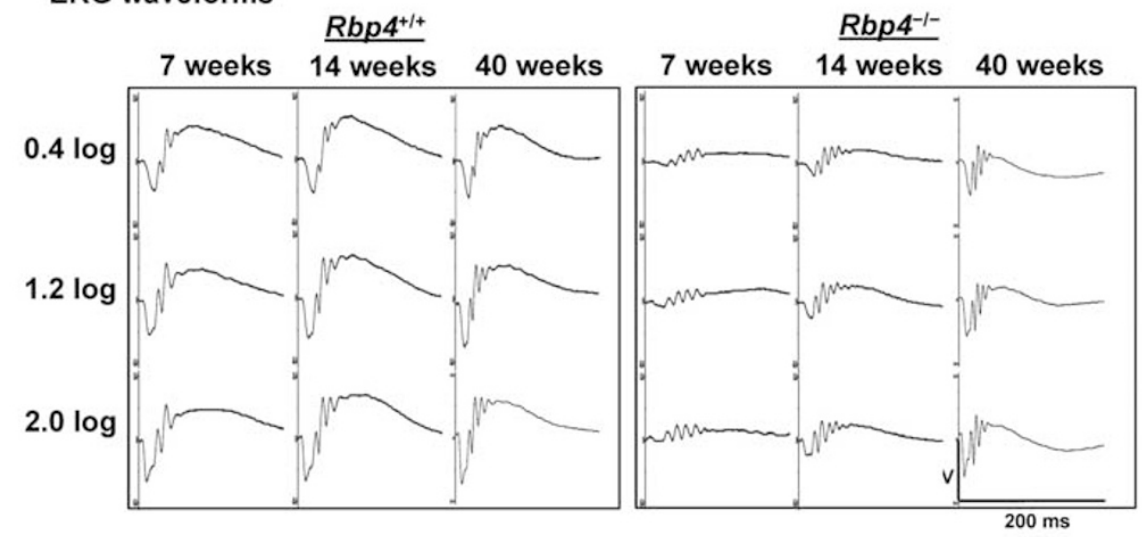

b ERG responses
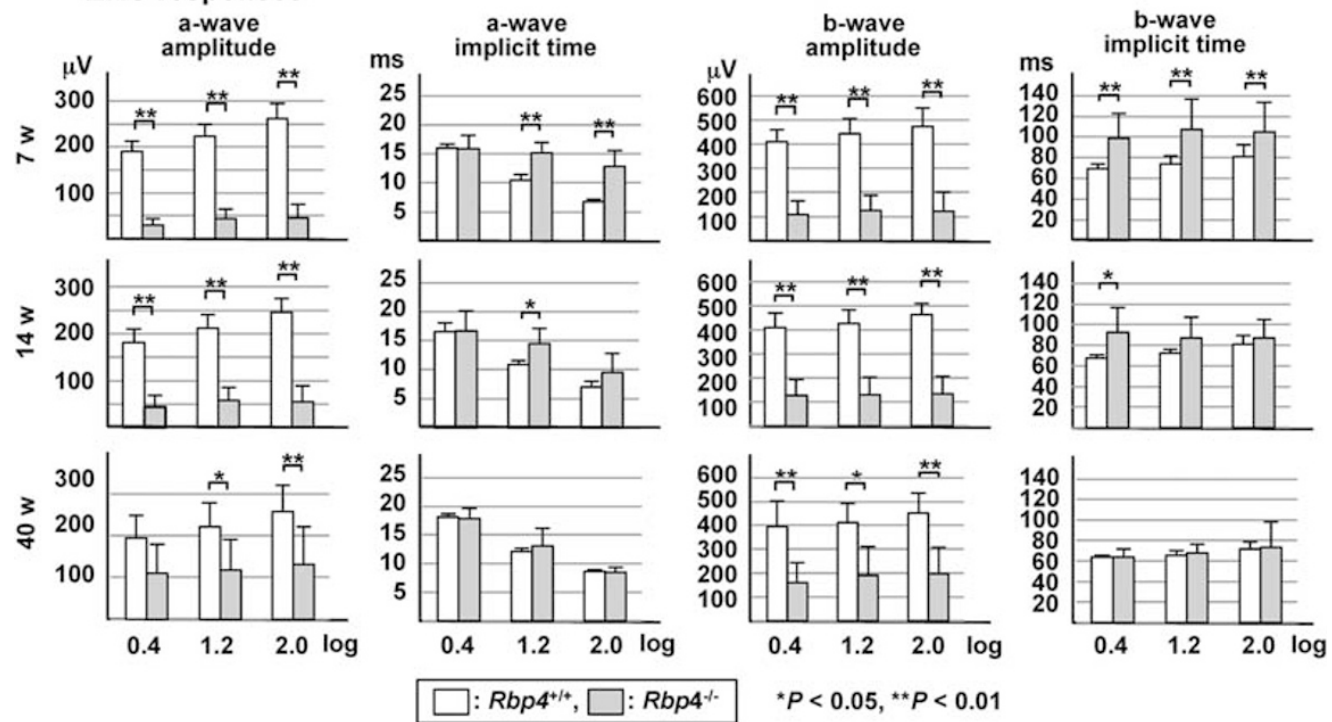

Figure 3 Abnormal retinal function. (a) Representative electroretinogram (ERG) waveforms. ERG responses in $R b p 4^{-/-}$mice had recovered moderately at 40 weeks of age, but were still lower compared with those in $R b p 4^{+/+}$mice. (b) ERG responses to varying light intensity. The a- and b-wave amplitudes were strongly reduced in $\mathrm{Rbp}^{-/-}$mice during the 40-week observation period. The a- and b-wave implicit times were significantly shorter in $R b p 4^{-/-}$ mice than at 7 weeks of age, but had recovered to normal by 40 weeks of age.

Quetol-812 resin (Nisshin EM, Tokyo, Japan) for $1 \mathrm{~h}$; the tube was kept open overnight to evaporate PO. The samples were transferred to fresh $100 \%$ resin and polymerized at $60^{\circ} \mathrm{C}$ for $48 \mathrm{~h}$. Ultrathin sections $(70 \mathrm{~nm})$ were cut with a diamond knife using an ultramicrotome (Ultracut UCT; Leica, Vienna, Austria) and mounted on copper grids. They were stained with $2 \%$ uranyl acetate at room temperature for $15 \mathrm{~min}$, washed with distilled water, and stained with lead stain solution (Sigma-Aldrich, Tokyo, Japan) at room temperature for $3 \mathrm{~min}$. The grids were examined under a transmission electron microscope (JEM-1400Plus; JEOL, Tokyo, Japan) at an acceleration voltage of $80 \mathrm{kV}$. Digital images $(2048 \times 2048$ pixels $)$ were taken with a CCD camera (Veleta; Olympus Soft lmaging Solutions GmbH, Münster, Germany).

\section{Funduscopic Examination}

Pupils were dilated with $0.5 \%$ tropicamide and $0.5 \%$ phenylephrine (Santen Pharmaceutical, Osaka, Japan) diluted 5- to 7.5-fold with physiological saline. Fundi were examined by indirect ophthalmoscopy and photographs were taken with a Genesis hand-held funduscope camera (Kowa, Nagoya, Japan) with a Volk 90D condensing lens (Volk Optical, Mentor, OH, USA) as described previously. ${ }^{20}$ The camera was fitted to a dissection microscope base for stability and a foot pedal was used to operate the shutter. Photographs of conscious mice were taken.

\section{Genotyping of the Crb1 Locus}

DNA samples isolated from tail biopsies were amplified separately for the wild-type allele and the mutant $r d 8$ allele as 

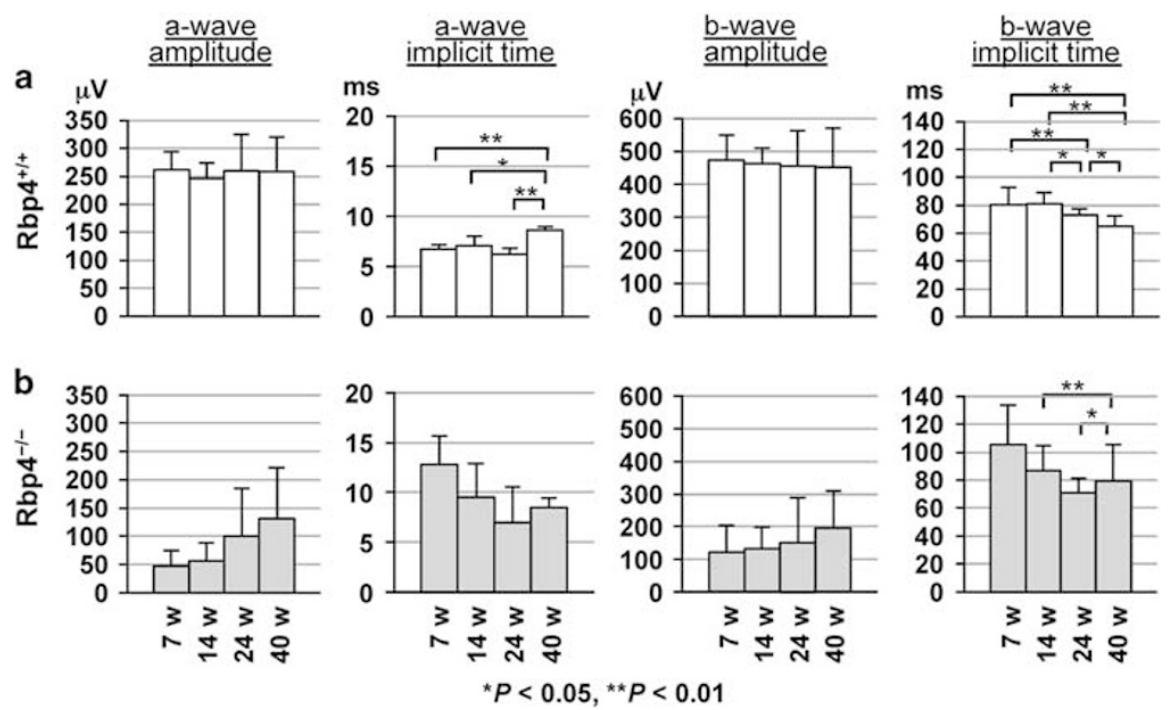

Figure 4 Electroretinogram (ERG) changes during growth. (a) ERG response in $R b p 4^{+/+}$mice. (b) ERG response in $R b p 4^{-/-}$mice. In $R b p 4^{-/-}$mice, the $\mathrm{a}$ - and b-wave amplitudes had not recovered to within the normal range even at 40 weeks of age.

described previously; ${ }^{3,17}$ the size of the amplified wild-type allele was $220 \mathrm{bp}$ and the mutant $r d 8$ allele was $244 \mathrm{bp}$.

\section{Determination of Serum and Liver Retinol Concentrations}

Serum and liver retinol concentrations were determined by high-performance liquid chromatography when the mice were 8 and 30 weeks of age. SRL determined serum retinol as described. ${ }^{22}$ Japan Food Research Company determined liver retinol as described. ${ }^{23}$ As retinyl esters are converted to retinol in this method, the amount of retinol was expressed as a total retinol.

\section{Number of Mice Used for Each Experiment}

Number of mice used for each experiment was shown in Supplementary Table S1.

\section{Statistical Analysis}

At least three independent experiments were carried out. Unpaired Student's $t$-tests were used for the statistical analysis. Results are expressed as mean \pm s.e. $P<0.05$ was considered to indicate a significant difference.

\section{RESULTS}

\section{Establishment of ES Cell Clones and Mouse Strains with} the Targeted Null Allele

After electroporation with the targeting vectors, 98 neo-resistant clones were screened for targeted recombination by using Southern blot analysis. With a neo probe, three clones (nos. 19, 83, and 87) gave a 5.1-kb band when digested with XhoI/SalI and an 8.8-kb band when digested with AgeI/HpaI (Supplementary Figure S1). All three clones digested with MunI gave 8.2- and 10.1-kb bands with the $5^{\prime}$ probe; the same clones digested with SacII/HpaI gave 7.5- and 9.3-kb bands with the $3^{\prime}$ probe (Supplementary Figure S1). These patterns indicated that the presence of the targeted allele. Clones 19 and 87 showed germline transmission and were used to create the homozygous mutant mice $\left(R b p 4^{-I-}\right)$. These mice were born at the expected Mendelian frequency, indicating that $R b p 4$ is not essential for normal prenatal development with a diet containing $1515 \mathrm{IU} / 100 \mathrm{~g}$ food. $R b p 4^{-/-}$adults were viable and fertile.

\section{Confirmation of the Null Mutation in $\mathbf{R b p 4}^{-/-}$Mice}

No $R b p 4$ mRNA was detected in the brain, lung, liver, and kidney of $R b p 4^{-/-}$mice by using RT-PCR (Figure 2a). Northern blots of liver RNA and western blots of serum confirmed the absence of Rbp4 mRNA (Figure 2b) and RBP4 protein (Figure 2c), respectively, in these mice.

\section{Retinal Function}

A long-term ERG study was performed to assess agedependent changes in retinal function. Representative ERG waveforms are shown in Figure 3 a.

The a- and b-wave amplitudes were severely reduced in $R b p 4^{-1-}$ mice throughout the 40 -week observation period. The a- and b-wave implicit times were longer in $R b p 4^{-1-}$ mice at 7 weeks of age, but recovered to normal at 40 weeks of age. Both strains showed increased a- and b-wave amplitudes and decreased a-wave implicit time with increased light intensity (Figure 3b).

To analyze the ERG changes during growth, the same data were plotted against mouse age (Figure 4). In $R b p 4^{+/+}$mice, there were almost no changes in the intensities of the a- and b-wave amplitudes with age. The a-wave implicit time was a little longer and the b-wave implicit time was a little shorter at 40 weeks than at 7 weeks of age (Figure $4 \mathrm{a}$ ); however, the reasons for this are unclear. Both the a- and b-wave 

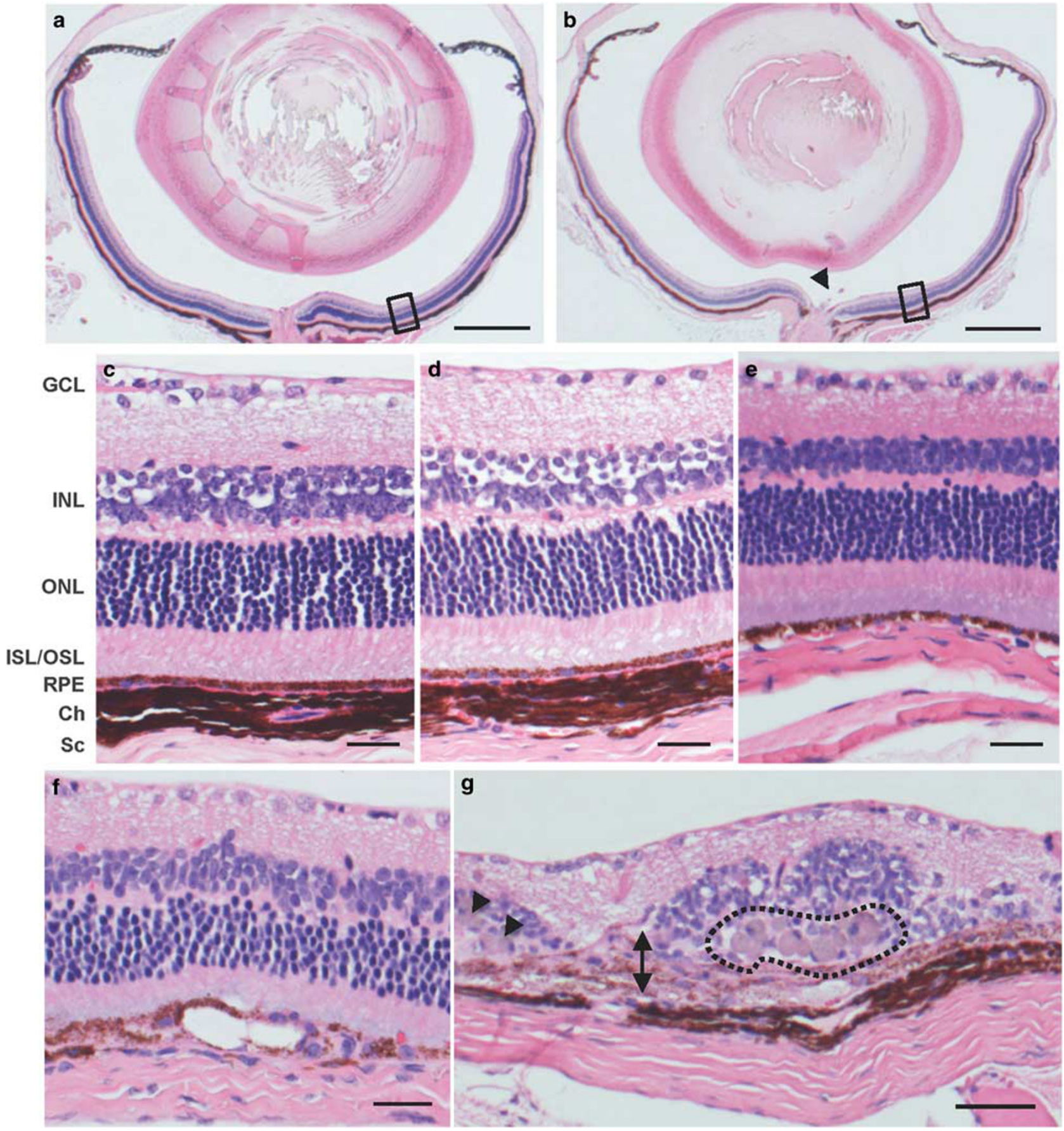

Figure 5 Histological analysis of the retina. Representative hematoxylin and eosin-stained sections of the retinas of 40-week-old mice at low (a and b) and high magnifications (c and $\mathbf{d}$ ) and lesions observed in the $R b p 4^{-/-}$retinas (e-i). (a) The entire retina of an $R b p 4^{+/+}$mouse. (b) The entire retina of an $R b p 4^{-1-}$ mouse. A hyaloid artery remnant extended from the optic disc towards the vitreous humor (arrowhead). (c) Central retina: the boxed area in (a) at higher magnification. (d) Central retina: the boxed area in (b) at higher magnification. (e) A representative section of an Rbp4 ${ }^{-/-}$peripheral retina showing choroidal loss. (f) Vacuolation in the retinal pigment epithelium. (g) Hypopigmented retinal pigment cells (arrow) or focal loss of the photoreceptor layer with appearance of large, irregular, macrophage-like cells (arrowheads and the areas bounded by a dashed line). (h) Focal loss of the photoreceptor layer and appearance of large, irregular, macrophage-like cells (arrowheads). (i) Focal collapse of the retinal structure with disappearance of the retinal and choroidal layers. Ch, choroid; GCL, ganglion cell layer; INL, inner nuclear layer; IS/OS, inner segments/outer segments; ONL, outer nuclear layer; RPE, retinal pigment epithelium; Sc, sclera. Scale bar: $500 \mu \mathrm{m}$ in panels a and b, $25 \mu \mathrm{m}$ in panels c-f, $50 \mu \mathrm{m}$ in panels $\mathrm{g}-\mathrm{I}$. 

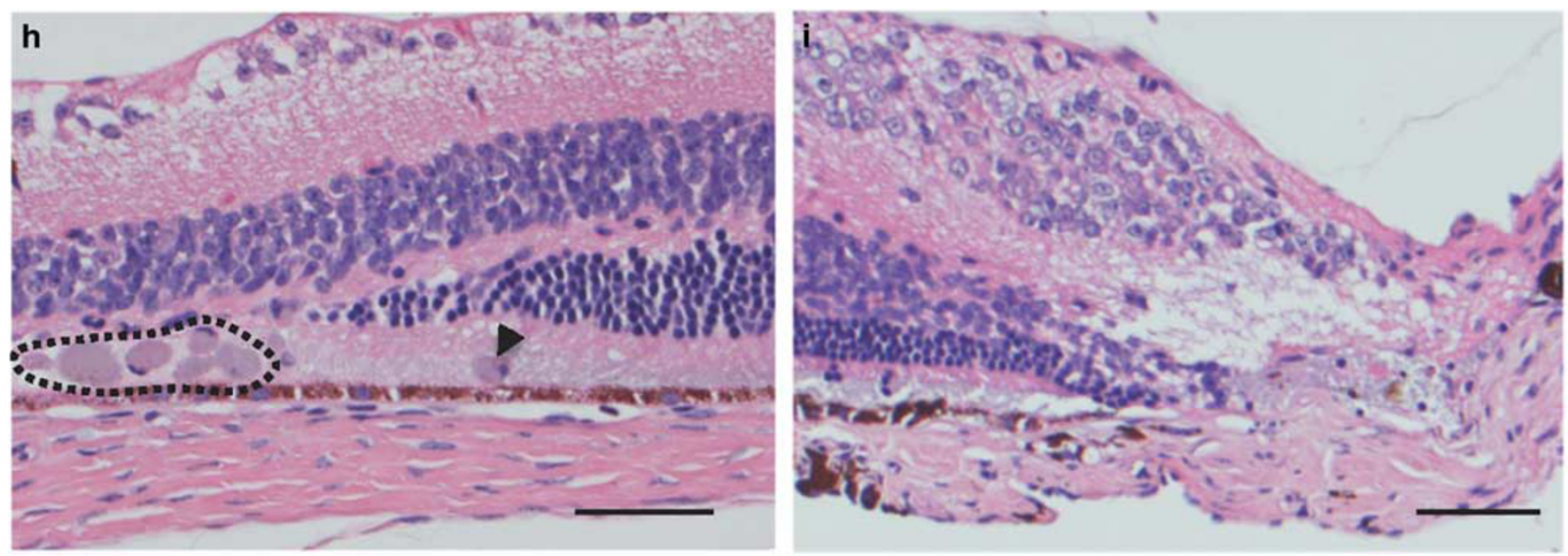

Figure 5 Continued

amplitudes were severely reduced in $R b p 4^{-1-}$ mice throughout the 40-week observation period. However, the delays in $\mathrm{a}$ - and b-wave implicit times were slight at 7 weeks and were within the normal range at 14 weeks (Figure $4 \mathrm{~b}$ ).

\section{Histological Comparison of the Retinas of $\mathrm{Rbp}^{+/+}$and Rbp4 ${ }^{-1-}$ Mice}

We examined whether the impairment of retinal function was caused by pathologic changes in the eyes. The entire retina of an $R b p 4^{+/+}$and an $R b p 4^{-/-}$were shown in Figures $5 \mathrm{a}$ and b and Supplementary Figure S2. A hyaloid artery remnant extended from the optic disc towards the vitreous humor (arrowhead) (Figure 5b). There were no obvious differences in the central retinas of $R b p 4^{+/+}$and $R b p 4^{-1-}$ mice (Figures $5 \mathrm{c}$ and $\mathrm{d}$ ); however, the peripheral retinas of most $R b p 4^{-1-}$ mice showed choroidal loss (5/7 eyes; Figure 5e). Furthermore, the $R b p 4^{-1-}$ peripheral retina showed various morphological defects (Figures $5 \mathrm{f}-\mathrm{i})$. Vacuolation (2/7 eyes) (Figure 5f) and formation of multiple layers of hypopigmented retinal pigment cells (2/7 eyes) (Figure $5 \mathrm{~g}$ ) were observed in the $R b p 4^{-1-}$ retinal pigment epithelium. Large, irregular, macrophage-like cells (3/7 eyes) ${ }^{24}$ appeared between the INL and retinal pigment epithelium in the regions where severe photoreceptor loss developed (2/7 eyes) (Figures $5 \mathrm{~g}$ and $\mathrm{h}$ ). One of the $R b p 4^{-1-}$ mice had retinal structure collapse including disappearances of both retinal and choroidal layers in the peripheral retina (Figure 5i).

\section{Measurement of Retinal Properties}

To evaluate the quantitative differences in the retinas from the $R b p 4^{+/+}$and $R b p 4^{-/-}$mice, various analyses were performed. The number of neurons in the ganglion cell layer and the thicknesses of the ONL, OSL, and whole retina were decreased in the peripheral region of retina from $R b p 4^{-1-}$ mice (Figure 6).

\section{Transmission Electron Microscopy}

Similar to histological analyses, transmission electron microscopy showed that $R b p 4^{-1-}$ mice had thinner photoreceptor layer, loss of retinal pigment cell, and thinner choroidal layer in the peripheral region (data not shown). We also found some abnormalities in the central retina. In $\mathrm{Rbp}^{-/-}$mice, the distance between the inner limiting membrane and outer plexiform layer was much shorter than that in $R b p 4^{+/+}$mice (Figure 7a), with fewer retinal ganglion cells (Figure 7b). Much fewer synapses were found in the inner plexiform layer (Figure 7b). Electron density in the outer plexiform layer (a layer of neuronal synapses) was very low in $R b p 4^{-1-}$ mice (Figure 7c). Although the number of mitochondria in photoreceptor cell of $R b p 4^{-1-}$ mice and $R b p 4^{+/+}$mice was similar, there were very few cristas in $R b p 4^{-1-}$ mice (Figure 7d). In addition, the Golgi apparatus was swollen (Figure 7e).

\section{Defects in the Ocular Fundus}

We examined the ocular fundus by indirect ophthalmoscopy. Developmental defects such as persistent hyaloid artery, retinal depigmentation, and optic disc abnormalities were found in $\mathrm{Rbp}^{-1-}$ mice (Figure 8). These defects did not recover by 40 weeks of age (Figure 8 ).

\section{Rd8 Mutation}

As reported by Mattapallil et al, ${ }^{17}$ the $r d 8$ mutation in the $\mathrm{Crb} 1$ gene is found in the C57BL/6N substrain but not in the C57BL/6J substrain. This mutation results in ocular spots and retinal lesions such as retinal folding, dysplasia of the nuclear layers, retinal degeneration, and vacuolation of the retinal pigment epithelium. To rule out this possibility that the defects we observed in $R b p 4^{-1-}$ mice were caused by the $r d 8$ mutation, we analyzed this mutation in our mice. C57BL/6N mice were obtained from Clea, or F1 hybrid between C57BL/ $6 \mathrm{~J}$ and $\mathrm{C} 57 \mathrm{BL} / 6 \mathrm{~N}$ carried the $r d 8$ mutation as expected (Figure 9a). However, we confirmed that none of the mice 
a

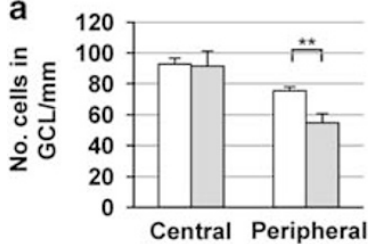

C

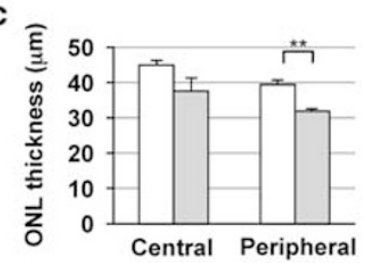

e

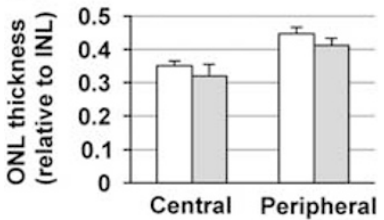

g

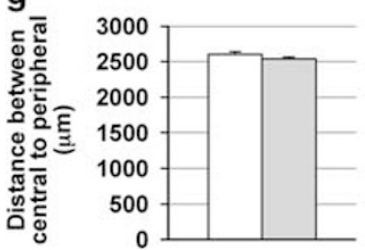

b

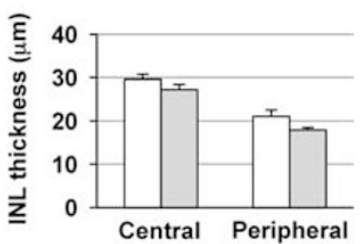

d

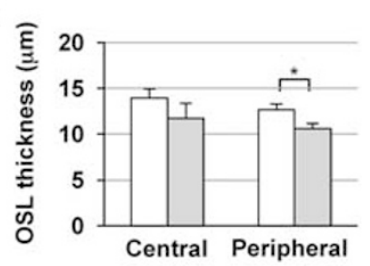

f
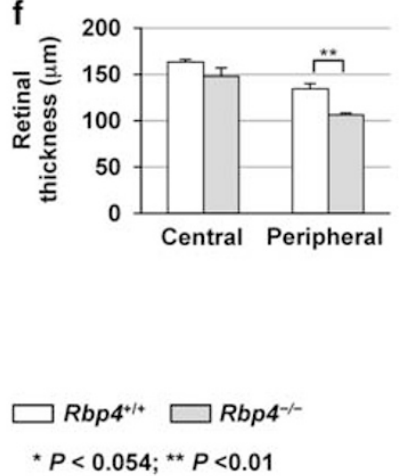

Figure 6 Quantitative comparison of retinal properties. (a) Cell number in the ganglion cell layer (GCL). Significant retinal ganglion cell loss was observed in the peripheral region of retinas from $R b p 4^{-1-}$ mice. (b) Thickness of the inner nuclear layer (INL). No significant differences were observed. (c) Thickness of the outer nuclear layer (ONL). The ONL in peripheral retinas was significantly thinner in $\mathrm{Rbp}^{-1-}$ mice than in $R b p 4^{+/+}$mice. (d) Thickness of the outer segment layer (OSL). The OSL in peripheral retinas was significantly thinner in $R b p 4^{-1-}$ mice than in $R b p 4^{+/+}$mice. (e) ONL/INL ratio. No significant differences were observed. (f) Retinal thickness from GCL to OSL. Retinal thickness was significantly decreased in the peripheral retinas of $R b p 4^{-/-}$mice. (g) Distance between central and peripheral retina. No significant difference was observed. Data are presented as means \pm s.e.

used in our morphological and functional analyses of the retina did so (Figure 9b).

\section{Retinol Concentration in the Plasma and Liver}

As various eye defects are caused by vitamin A deficiency, ${ }^{25}$ we determined retinol concentrations in the plasma and liver. Serum retinol concentration in $R b p 4^{+/+}$mice was $48.5 \pm 14.3$ at 8 weeks of age and $97 \pm 3.6 \mathrm{IU} / \mathrm{dl}$ at 30 weeks (Figure 10a). As expected, serum retinol in $R b p 4^{-1-}$ mice was below the detection threshold $(<5 \mathrm{IU} / \mathrm{dl})$ (Figure 10a).

Liver total retinol content at 8 weeks was $58.2 \pm 5.2 \mathrm{mg} /$ $100 \mathrm{~g}$ in $R b p 4^{+/+}$and $58.3 \pm 4.0 \mathrm{mg} / 100 \mathrm{~g}$ in $R b p 4^{-1-}$ mice (Figure 10b). Thus, liver total retinol content was similar in the two strains, suggesting that the absence of RBP4 markedly reduces serum retinol levels, but does not impair accumulation of hepatic retinol stores at this age. At 30 weeks, liver total retinol content was $153.3 \pm 2.9 \mathrm{mg} / 100 \mathrm{~g}$ in $R b p 4^{+/+}$and $188.8 \pm 5.6 \mathrm{mg} / 100 \mathrm{~g}$ in $R b p 4^{-1-}$ mice (Figure 10b). This difference at 30 weeks indicated an inverse relation between the liver retinol content and serum RBP4 concentration. It is of interest that liver total retinol concentration was increased with age even in $R b p 4^{+/+}$mice.

\section{Time Course of Rbp4 Expression}

To examine whether the increase in serum retinol concentration with age in $R b p 4^{+/+}$mice resulted from an increased expression of Rbp4 in the liver, we examined the time course of Rbp4 mRNA expression by using qRT-PCR. The level of Rbp 4 mRNA at 12 and 24 weeks of age was $133 \%$ and $131 \%$, respectively, of the level at 6 weeks (Figure 11a). The difference between 6 and each of the later time points was significant; the absence of a significant difference between 12 and 24 weeks suggested that Rbp 4 mRNA expression in the liver reached a maximum level by 12 weeks.

At 12 and 24 weeks, the serum level of RBP 4 was $276 \%$ and $304 \%$, respectively, of the level at 6 weeks (Figure 11b). Again, there was a significant difference between 6 weeks and each of the two other time points, but not between 12 and 24 weeks. These results are consistent with those for mRNA expression levels in the liver in that the maximum was reached at 12 weeks. These data suggest that the increase in serum retinol with age is due to the increase in $R b p 4$ mRNA expression.

\section{DISCUSSION}

Here, we established that $R b p 4^{-1-}$ mice in the C57BL/6 background show much more severe phenotypes in terms of ERG abnormalities than $R b p 4^{-1-}$ mice reported previously. ${ }^{15}$ In accordance with these abnormalities, structural changes were observed, such as loss of photoreceptor and choroidal cells, fewer synapses in the inner plexiform layer, and the shorter distance between the inner limiting membrane and the outer plexiform layer with fewer ganglion cells. Furthermore, our mice had unexpected developmental ocular abnormalities, such as persistant hyaloid artery, retinal depigmentation, and persistent optic disc abnormality.

Quadro et $a l^{15}$ found that $R b p 4^{-1-}$ mice had impaired visual function, as evidenced by abnormal ERG. They had decreased sensitivity to light only in the b-wave amplitude, with progressive improvement such that this sensitivity approached that of wild-type mice by 24 weeks of age; no abnormalities in other parameters were reported. Our $R b p 4^{-1-}$ mice showed decreased sensitivity in both a- and b-wave amplitudes, and retinal impairment did not recover completely, even at 40 weeks of age. Thus, our mice showed more severe retinal impairment than the mice described by Quadro et al. ${ }^{15}$ 


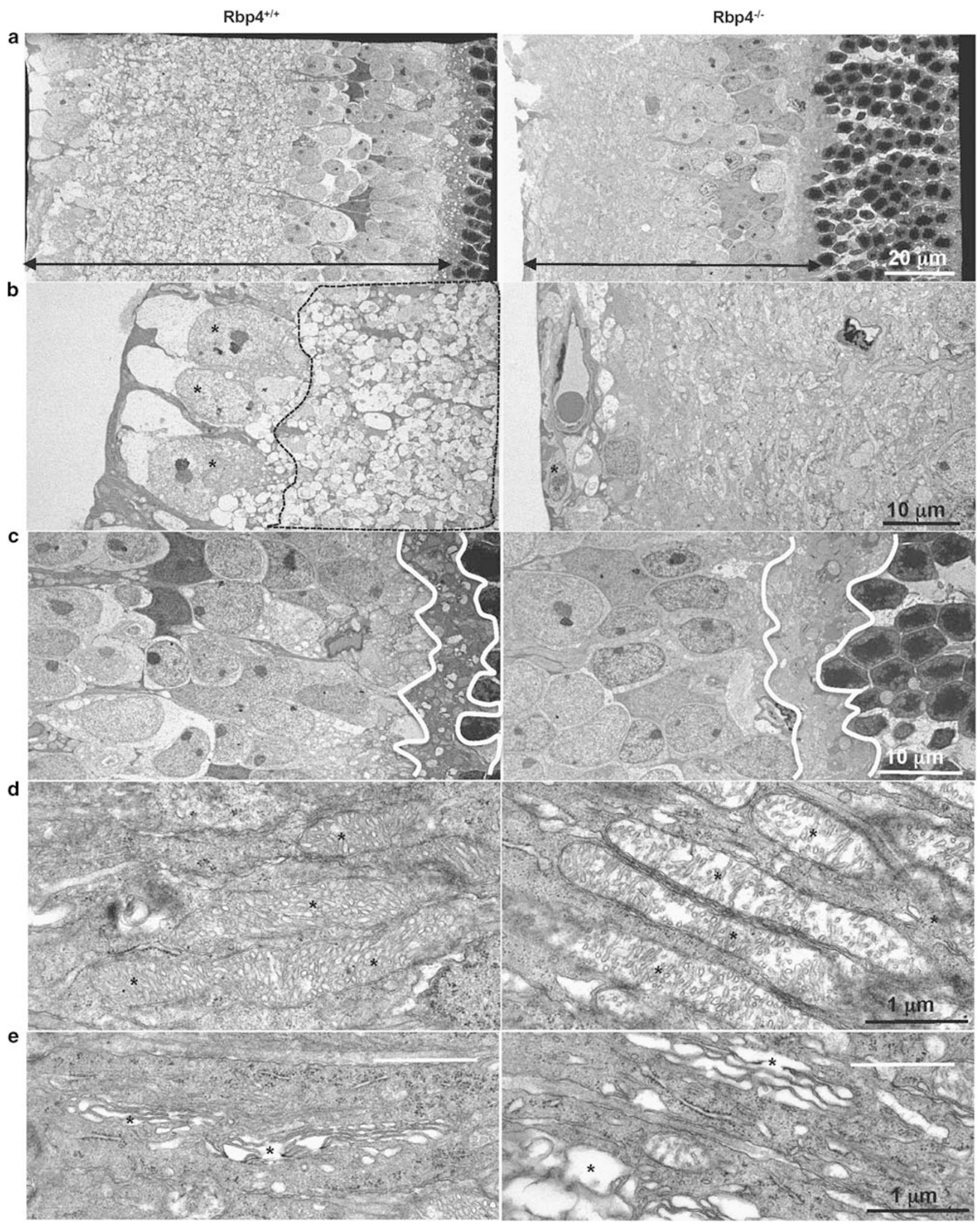

Figure 7 Transmission electron microscopy. (a) Distance between the inner limiting membrane and outer plexiform layer (↔口). This distance was much

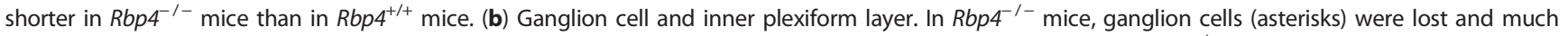
fewer synapses were found in inner plexiform layer (bounded by a dashed line $\square$ ). (c) Outer plexiform layer. In Rbp ${ }^{-/-}$mice, electron density in the outer plexiform layer (surrounded by white line) was very low. (d) Mitochondria in a photoreceptor cell. Although the number of mitochondria (asterisks) was similar in $\mathrm{Rbp}^{-/-}$and $\mathrm{Rbp}^{+/+}$mice, there were very few cristas in $R b p 4^{-/-}$mice. (e) Golgi apparatus (asterisks) was swollen in $R b p 4^{-/-}$mice. 


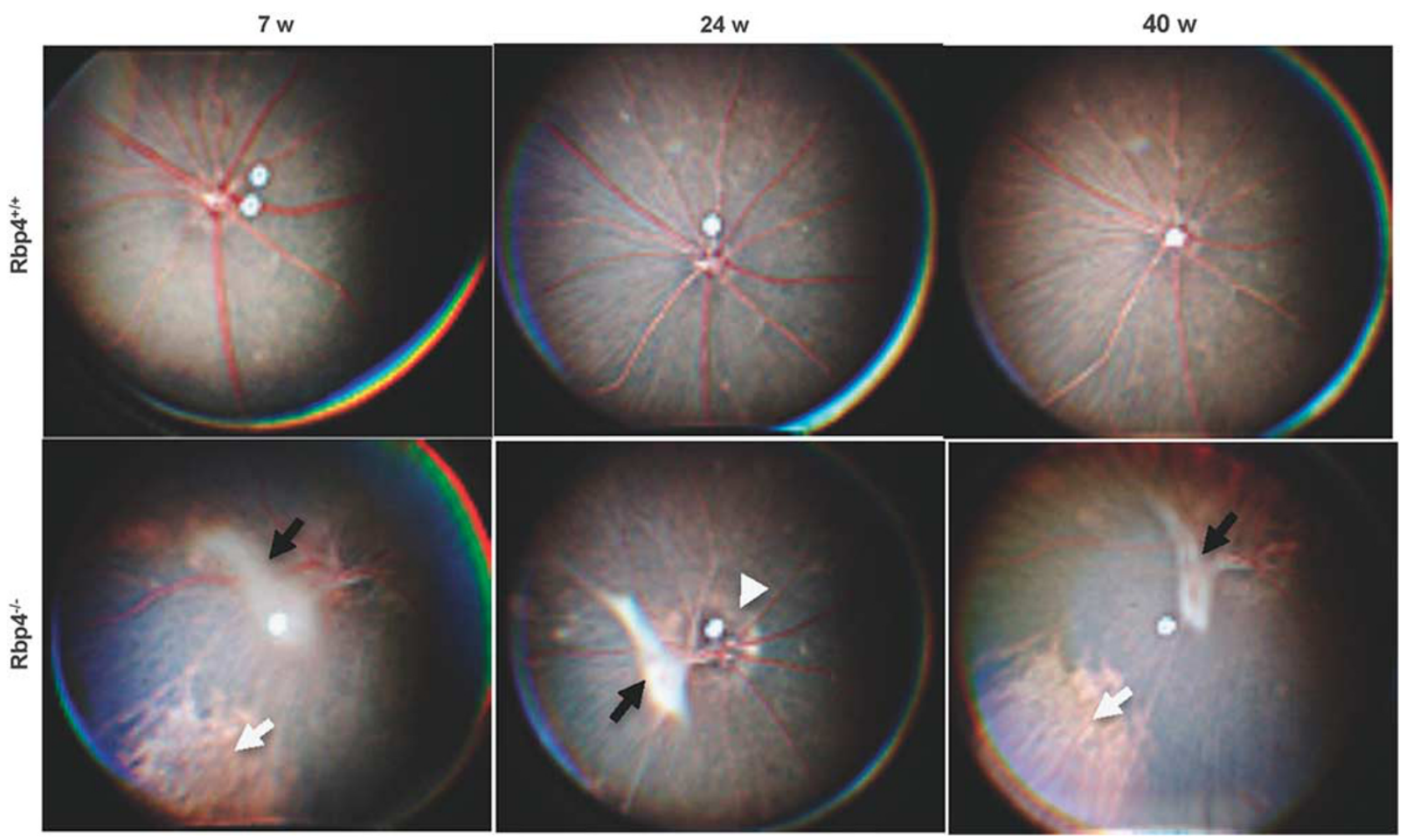

Figure 8 Defects in the ocular fundus. Persistent hyaloid artery, retinal depigmentation, and optic disc abnormalities were observed as early as 7 weeks of age in $\mathrm{Rbp4}^{-/-}$mice. Black arrow: hyaloid artery; white arrow: retinal depigmentation; white arrowhead: optic disc abnormality.
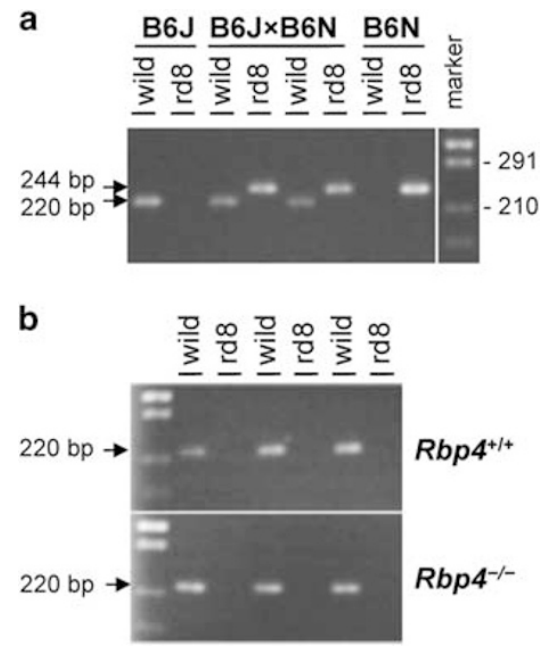

Figure 9 Absence of the mutant $r d 8$ allele of the Crb1 gene. Representative gels show the wild-type (220 bp) and $r d 8$ (244 bp) bands. (a) The mutant $r d 8$ allele was detected in C57BL/6N (B6N) but not in

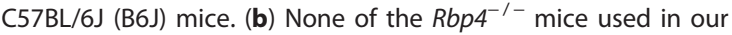
morphological and functional assessment of the retina carried the $r d 8$ mutation.

As the $r d 8$ mutation was not observed in our mice, retinal impairment could be caused either by retinol deficiency in the blood or by loss of $R b p 4$ expression in the eyes. Rbp4 is expressed in the retinal pigment epithelium. ${ }^{26-29}$ However, little is known about the function of RBP4 in the eye. Quadro $e t a l^{30}$ generated a transgenic mouse strain (hRBP4: $\mathrm{mRbp}^{-{ }^{-}}$) that expressed human RBP4 under the control of the muscle creatine kinase promoter in the $R b p 4^{-/-}$background. Although no human RBP4 was detected in the retinal pigment epithelium of hRBP4: $\mathrm{mRbp} 4^{-/-}$mice, a normal ERG profile and normal retinol levels were observed in the eyes. These data suggest that circulating RBP4 efficiently delivers retinol to the eye, and that RBP4 present in the eye appears to have no role in maintaining the visual function. Thus, ocular phenotypes in our $R b p 4^{-/-}$mice may be caused by loss of circulating RBP4 and retinol. In other words, deficiency of circulating retinol may cause abnormalities of photoreceptor cells and synapses among ganglion cells and Muller cells/bipolar cells, because the a- and b-wave amplitude and implicit time are indicators of the functions of these cells.

In human patients with no detectable serum RBP4 because of RBP4 gene mutations, atrophy of the retinal pigment epithelium and iris coloboma are common. ${ }^{31,32}$ Although iris coloboma was not observed in our $R b p 4^{-1-}$ mice, retinal depigmentation in these mice might be similar to atrophy of the pigment epithelium in humans with RBP4 gene mutations. In contrast, Quadro et al ${ }^{15}$ did not observe such ocular abnormalities in their histological analysis under a light or electron microscope. Our $R b p 4^{-1-}$ mice have the C57BL/6 genetic background as confirmed by using 123 polymorphic 
a

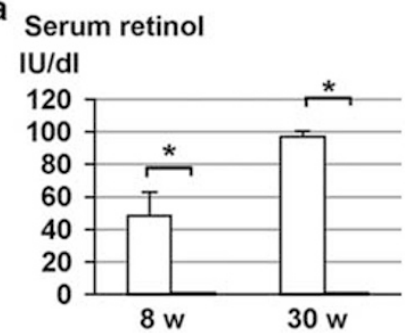

b

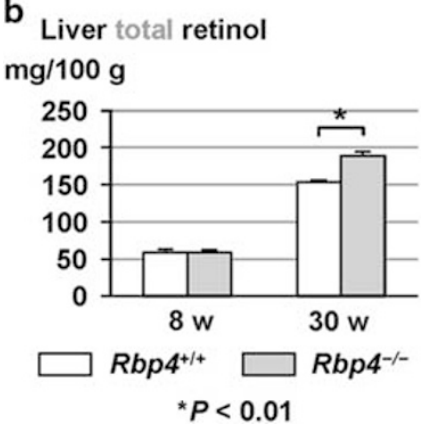

Figure 10 Retinol concentration. (a) Serum retinol concentration. Serum retinol was not detected in $R b p 4^{-1-}$ mice. (b) Liver total retinol content. In both strains, retinol accumulated in the liver by 30 weeks of age.

markers, whereas Quadro et al ${ }^{15}$ used mice in a mixed background (129xC57BL/6 J). This strain difference might be the reason for the more severe phenotypes of our $R b p 4^{-1-}$ mice compared with those of the mice studied by Quadro et al.

In $R b p 4^{-1-}$ mice, liver retinol content was the same as that in $\mathrm{Rbp}^{+/+}$mice at 8 weeks, but became higher than that in $R b p 4^{+/+}$mice at 30 weeks. This is consistent with the previous findings ${ }^{15,33}$ that there was no significant difference in total hepatic retinol between $R b p 4^{-1-}$ and $R b p 4^{+/+}$mice at 3 and 13 weeks of age, and a significantly higher hepatic retinol level in $R b p 4^{-/-}$compared with that in $R b p 4^{+/+}$mice at later stages. In the present study, we found that the amount of retinol in the liver was increased, even in $R b p 4^{+/+}$mice, at 30 weeks compared with at 8 weeks of age. Quadro et al ${ }^{15}$ examined individual levels for hepatic retinol $(28 \mu \mathrm{g} / \mathrm{g})$ and hepatic retinyl ester levels $(1800 \mu \mathrm{g} / \mathrm{g})$. As the average mol\% for retinol in retinyl esters is 0.53 , hepatic retinyl ester levels were equivalent to $954 \mu \mathrm{g} / \mathrm{g}$ retinol. Thus, the liver total retinol level in their mice was $982 \mu \mathrm{g} / \mathrm{g}(954 \mu \mathrm{g} / \mathrm{g}+28 \mu \mathrm{g} / \mathrm{g})$ and was higher compared with our mice $(583 \mu \mathrm{g} / \mathrm{g})$. This is in good agreement with the fact that Quadro et al ${ }^{15}$ used a diet containing $0.66 \mathrm{mg} / 100 \mathrm{~g}$, which is $\sim 145 \%$ of the retinol than the one used in our study $(0.454 \mathrm{mg} / 100 \mathrm{~g})$.

Structural variation is widespread in mammalian genomes $^{34,35}$ and is an important cause of disease. ${ }^{36}$ Genome-wide association studies identified single-nucleotide polymorphisms in several genes, such as GRIA4, KCNQ5, RDH5, LAMA2, BMP2, SIX6, PRSS56, GJD2, RASGRF1, $Z C 3 H 11 B$, and $W N T 7 B$, as risk factors for refractive a Rbp4 mRNA expression in liver

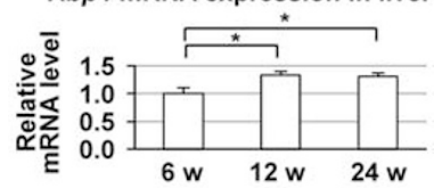

b Serum RBP4

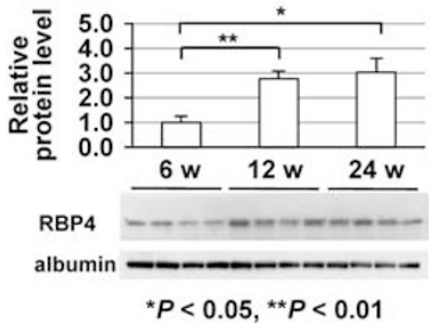

Figure 11 Rbp4 expression and retinol concentration. (a) Expression of Rbp4 mRNA in the liver assessed by means of quantitative reverse transcription-polymerase chain reaction (qRT-PCR). Expression level at 6 weeks of age was set as 1.0. (b) Serum level of RBP4 protein. Expression profiles of Rbp4 in the liver and serum were similar.

error including myopia. ${ }^{6-8}$ Although some of these genes (SIX6, PRSS56, and WNT7B) function in eye development, ${ }^{7,8}$ it remains unclear how such sequence variants shape phenotypic variation. Phenotyping studies by using genetically engineered mice with different genetic backgrounds in conjunction with sequence analyses will provide useful information on the association between genomic and phenotypic variations.

Supplementary Information accompanies the paper on the Laboratory Investigation website (http://www.laboratoryinvestigation.org)

\section{ACKNOWLEDGMENTS}

We thank Kyoko Ikeda and Shundan Jin for their technical assistance. This work was supported by a Grant-in-Aid for Scientific Research (C) (grant number 24590404; Japan Society for the Promotion of Science) (to ZL), and Grant-in-Aid for Scientific Research (S) (grant number 21220010; Japan Society for the Promotion of Science), Scientific Support Programs for Cancer Research Grant-in-Aid for Scientific Research on Innovative Areas (grant number 221S0001; Ministry of Education, Culture, Sports, Science, and Technology), CREST (JST), and a research grant from TransGenic (to KY).

\section{DISCLOSURE/CONFLICT OF INTEREST}

The authors declare no conflict of interest.

1. Episkopou V, Maeda S, Nishiguchi $\mathrm{S}$ et al. Disruption of the transthyretin gene results in mice with depressed levels of plasma retinol and thyroid hormone. Proc Natl Acad Sci USA 1993;90: 2375-2379.

2. Goodman DS. Plasma Retinol-Binding Protein. Academic Press: New York, NY, 1984.

3. Mehalow AK, Kameya S, Smith RS et al. CRB1 is essential for external limiting membrane integrity and photoreceptor morphogenesis in the mammalian retina. Hum Mol Genet 2003;12:2179-2189.

4. Soprano DR, Blaner WS. Plasma retinol-binding protein. In: Sporn MB, Roberts $A B$, Goodman DS (eds). The Retinoids: Biology, Chemistry, and Medicine, 2nd edn. Raven Press: New York, NY, USA, 1994, pp 257-281. 
5. Vogel S, Gamble MV, Blaner WS. Biosynthesis, Absorption, Metabolism and Transport of Retinoids. Springer: Berlin, Germany, 1999.

6. Fan $\mathrm{Q}$, Barathi VA, Cheng CY et al. Genetic variants on chromosome 1 q41 influence ocular axial length and high myopia. PLoS Genet 2012;8:e1002753.

7. Miyake $M$, Yamashiro $K$, Tabara $Y$ et al. Identification of myopiaassociated WNT7B polymorphisms provides insights into the mechanism underlying the development of myopia. Nat Commun 2015;6: 6689.

8. Verhoeven VJ, Hysi PG, Wojciechowski R et al. Genome-wide metaanalyses of multiancestry cohorts identify multiple new susceptibility loci for refractive error and myopia. Nat Genet 2013;45:314-318.

9. Beck JA, Lloyd S, Hafezparast M et al. Genealogies of mouse inbred strains. Nat Genet 2000;24:23-25.

10. Keane TM, Goodstadt L, Danecek $P$ et al. Mouse genomic variation and its effect on phenotypes and gene regulation. Nature 2011;477: 289-294.

11. Yalcin B, Wong K, Agam A et al. Sequence-based characterization of structural variation in the mouse genome. Nature 2011;477: 326-329.

12. Surwit RS, Kuhn CM, Cochrane $\mathrm{C}$ et al. Diet-induced type II diabetes in C57BL/6 J mice. Diabetes 1988;37:1163-1167.

13. Kobayashi M, Ohno T, Tsuchiya $T$ et al. Characterization of diabetesrelated traits in MSM and JF1 mice on high-fat diet. J Nutr Biochem 2004;15:614-621.

14. Wang J, Ohmuraya M, Suyama K et al. Relationship of strain-dependent susceptibility to experimentally induced acute pancreatitis with regulation of Prss1 and Spink3 expression. Lab Invest 2010;90: 654-664.

15. Quadro L, Blaner WS, Salchow DJ et al. Impaired retinal function and vitamin $A$ availability in mice lacking retinol-binding protein. EMBO J 1999;18:4633-4644.

16. Zhao G, Li Z, Araki K et al. Inconsistency between hepatic expression and serum concentration of transthyretin in mice humanized at the transthyretin locus. Genes Cells 2008;13:1257-1268.

17. Mattapallil MJ, Wawrousek EF, Chan CC et al. The Rd8 mutation of the Crb1 gene is present in vendor lines of C57BL/6N mice and embryonic stem cells, and confounds ocular induced mutant phenotypes. Invest Ophthalmol Vis Sci 2012;53:2921-2927.

18. Yagi T, Tokunaga T, Furuta $Y$ et al. A novel ES cell line, $\Pi 2$, with high germline-differentiating potency. Analytical Biochem 1993;214:70-76.

19. Li Z, Zhao G, Shen J et al. Enhanced expression of human CDNA by phosphoglycerate kinase promoter-puromycin cassette in the mouse transthyretin locus. Transgenic Res 2011;20:191-200.

20. Sato $H$, Suzuki $T$, lkeda $K$ et al. A monogenic dominant mutation in Rom1 generated by $\mathrm{N}$-ethyl- $\mathrm{N}$-nitrosourea mutagenesis causes retinal degeneration in mice. Mol Vis 2010;16:378-391.
21. Mi XS, Zhang $X$, Feng $Q$ et al. Progressive retinal degeneration in transgenic mice with overexpression of endothelin-1 in vascular endothelial cells. Invest Ophthalmol Vis Sci 2012;53:4842-4851.

22. Notsumoto S, Kobayashi K, Hirauchi K et al. Determination of retinol in serum by high-performance liquid chromatography with fluorometric detection using a column-switching system. Clin Chem (in Japanese) 1993;22:190-194.

23. Thompson LB, Schimpf KJ, Stiner LA et al. Determination of vitamin A (retinol) in infant and medical nutritional formulas with $A O A C$ method 992.06 using a modified extraction procedure: singlelaboratory validation. J AOAC Int 2010;93:1523-1529.

24. Luhmann UF, Robbie $S$, Munro PM et al. The drusen-like phenotype in aging Ccl2-knockout mice is caused by an accelerated accumulation of swollen autofluorescent subretinal macrophages. Invest Ophthalmol Vis Sci 2009;50:5934-5943.

25. Clagett-Dame M, Knutson D. Vitamin A in reproduction and development. Nutrients 2011;3:385-428.

26. Adler AJ, Edwards RB. Human interphotoreceptor matrix contains serum albumin and retinol-binding protein. Exp Eye Res 2000;70:227-234.

27. Herbert J, Cavallaro T, Martone R. The distribution of retinol-binding protein and its mRNA in the rat eye. Invest Ophthalmol Vis Sci 1991;32: 302-309.

28. Martone RL, Schon EA, Goodman DS et al. Retinol-binding protein is synthesized in the mammalian eye. Biochem Biophys Res Commun 1988;157:1078-1084.

29. Ong DE, Davis JT, O'Day WT et al. Synthesis and secretion of retinolbinding protein and transthyretin by cultured retinal pigment epithelium. Biochemistry 1994;33:1835-1842.

30. Quadro L, Blaner WS, Hamberger L et al. Muscle expression of human retinol-binding protein (RBP). Suppression of the visual defect of RBP knockout mice. J Biol Chem 2002;277:30191-30197.

31. Cukras C, Gaasterland T, Lee $P$ et al. Exome analysis identified a novel mutation in the RBP4 gene in a consanguineous pedigree with retinal dystrophy and developmental abnormalities. PLoS One 2012;7:e50205.

32. Seeliger MW, Biesalski HK, Wissinger B et al. Phenotype in retinol deficiency due to a hereditary defect in retinol binding protein synthesis. Invest Ophthalmol Vis Sci 1999;40:3-11.

33. Paik J, Vogel $S$, Quadro $L$ et al. Vitamin A: overlapping delivery pathways to tissues from the circulation. J Nutr 2004;134:276S-280S.

34. Mills RE, Walter K, Stewart C et al. Mapping copy number variation by population-scale genome sequencing. Nature 2011;470:59-65.

35. Quinlan AR, Clark RA, Sokolova $S$ et al. Genome-wide mapping and assembly of structural variant breakpoints in the mouse genome. Genome Res 2010;20:623-635.

36. Zhang Y, Martens JW, Yu JX et al. Copy number alterations that predict metastatic capability of human breast cancer. Cancer Res 2009;69: 3795-3801. 